\title{
Serum levels of anti-carbonic anhydrase antibodies and erythrocyte oxidative stress markers in endometriosis
}

\author{
Ulku Mete Ural ${ }^{1} \cdot$ Ahmet Alver $^{\mathbf{2}} \cdot$ Hasan $_{\mathrm{Efe}^{\mathbf{3}}} \cdot$ Ayse Senturk $^{\mathbf{2}} \cdot$ Imran Ince $^{\mathbf{2}} \cdot$ Beril Gurlek $^{\mathbf{1}}$ \\ ${ }^{\prime}$ Department of Obstetrics \& Gynecology, Recep Tayyip Erdogan University, Faculty of Medicine, Rize, Turkey \\ ${ }^{2}$ Department of Biochemistry, Karadeniz Technical University, Faculty of Medicine, Trabzon, Turkey \\ ${ }^{3}$ Department of Biochemistry, Recep Tayyip Erdogan University, Faculty of Medicine, Rize, Turkey
}

\section{ABSTRACT}

Aim: To evaluate the serum levels of anti-carbonic anhydrase I-II antibodies and erythrocyte oxidative stress markers in endometriosis.

Method: This case-control laboratory investigation was performed in the obstetrics and gynecology department of a tertiary center. Serum anti-carbonic anhydrase I and II antibodies and erythrocyte oxidative stress markers (superoxide dismutase, malondialdehyde, glutathione peroxidase and catalase) were compared with control group $(n=30)$ in the endometriosis group $(n=33)$. Correlation between carbonic anhydrase autoantibodies and oxidative stress markers were tested.

Results: Serum levels of anti-carbonic anhydrase II antibodies were found to be significantly increased in the endometriosis group compared to controls. The erythrocyte antioxidant enzyme activities (superoxide dismutase, glutathione peroxidase and catalase) and malondialdehyde levels in erythrocytes were increased in endometriosis group; but only glutathione peroxidase activity and malondialdehyde levels were significantly higher in endometriosis group. No correlation was detected between anti-carbonic anhydrase antibodies and oxidative stress markers.

Conclusions: Our results indicate that erythrocyte oxidative stress and anti-carbonic anhydrase antibodies may be involved in the pathophysiology of endometriosis.

Keywords: Endometriosis, carbonic anhydrase I, carbonic anhydrase II, antibodies, antioxidants.

(C) 2020 experimentalbiomedicalresearch.com

$\triangle$ Dr. Ulku Mete Ural,

Bolu Abant İzet Baysal University, School of Medicine

Department of Obstetrics \& Gynecology, Bolu, Turkey

E-mail: ulkumete2004@yahoo.com

Received: 2020-04-13 / Revisions: 2020-04-25

Accepted: 2020-04-29 / Publication Date: 2020-06-13

\section{Introduction}

Endometriosis is the ectopic growth of endometrial cells. It can be seen in about $10 \%$ of women of reproductive age [1]. The etiology of endometriosis has not been clarified yet. However, oxidative stress and malfunction of cellular and humoral immunity are possible mechanisms associated with the pathogenesis [2,3]. Endometriosis patients have diminished concentrations of antioxidant enzymes reminding that reduced peritoneal antioxidant levels may reflect an increase in the levels of reactive oxygen species (ROS) levels [2]. Recently, biochemical changes like oxidation 
of membranes and glutathione decrement have been reported in erythrocyte [4].

Antibodies directed against endometrial antigens and accumulation of complement system have been demonstrated in endometriosis patients. Antigens triggering such an immune response may develop against the human chorionic gonadotropin receptor, including the enzymes carbonic anhydrase (CA) I and II, CA 125 and transferrin in endometriosis $[3,5]$.

Carbonic anhydrases (CA; EC 4.2.1.1) activate the transformation of carbon dioxide and the bicarbonate ion. In mammals, sixteen isoenzymes of CA have been described and those have different tissue distribution and cellular localization. They participate in important metabolic reactions including respiration, transport of $\mathrm{CO} 2$ and bicarbonate, maintenance of carbon dioxide balance and $\mathrm{pH}$, and excretion of electrolytes [6]. Even though CA I and CA II exist in the epithelium of various organs, they are mainly expressed in erythrocyte. CA I activity is has a remarkably reduced action than CA II and thus, it is important the majority of the total CA activity. Recently, several publications demonstrated the pattern of autoimmune reaction against CA II in various pathologies [7-10]. The mechanism causing antibody creation has not been yet elucidated but oxidative stress is supposed to be involved in this etiopathogenesis [11].

In recent years, CA autoantibodies have been determined in some autoimmune diseases. To the best of our knowledge, the association between anti-CA antibodies (Anti-CAA) with antioxidant enzymes in endometriosis has not yet been tested in the medical literature (PubMed). Our aim was to analyze the levels of erythrocyte oxidative stress markers and antiCAA in endometriosis, and to seek whether there is a relationship between these indicators and discuss a possible role of anti-CAA in the pathogenesis of endometriosis.

\section{Materials and Methods}

This case-control laboratory investigation was done in the Obstetrics and Gynecology Department of a University Hospital after the approval of the local Institutional Review Board (Date and Decision no: 2014/04/17, 2014/65). The rights of all participants were protected and written informed consents were obtained before the study according to the Helsinki Declaration. This study included 33 women with endometriosis and 30 healthy women. The diagnosis of all patients with endometriosis was confirmed histologically after laparoscopy. Patients in the endometriosis group had at least stage 3 endometriosis. Control groups consisted of age-matched healthy, regularly menstruating women without pain, pelvic anomalies and previous history of pelvic surgery. In the past, they had at least one successful pregnancy.

Exclusion criteria consisted of a history of pregnancy loss, systemic diseases, smoking habit, use of any medications [such as oral contraceptives, hormonal drugs, steroids, aspirin] in the last 3 months and unwillingness for enrollment in the study. Serum levels of anti-carbonic anhydrase I and II antibodies and erythrocyte oxidative stress markers (superoxide dismutase, malondialdehyde, glutathione peroxidase, and catalase) were compared and the correlation between these indicators were tested.

Measurement of serum Anti-CAA I and Anti$\boldsymbol{C A A}$ II: Human erythrocyte CA I and CA II isoenzymes were purchased (Sigma Chemical Co., St. Louis, MO, USA). Serum anti-CAA II titers were determined by ELISA method as described in previous publication [8]. Briefly, 
ELISA plates (BD Biosciences, USA) were coated with CA-I and CA-II $(50 \mu \mathrm{L}$ of 10 $\mu \mathrm{g} / \mathrm{ml})$ in carbonate buffer $(0.05 \mathrm{mM}, \mathrm{pH}=9.6)$ and incubated overnight at $4{ }^{\circ} \mathrm{C}$. The coated plates were washed phosphate buffer $(\mathrm{pH}=7)$. Skimmed milk (2\%) in phosphate buffer was used for blocking. After washing process, incubation of the wells with $100 \mu \mathrm{L}$ of serum diluted with dilution buffer was carried out (1:200). Following washing, every well was incubated with $100 \mu \mathrm{L}$ of 1:2000 dilution of peroxidase-conjugated anti-human IgG antiserum (Sigma Chemical, St. Louis, MO, USA). After the final washing, ELISA wells were incubated with $100 \mu \mathrm{L} \quad$ ophenylenediamine solution and this reaction was quit by $100 \mu \mathrm{L}$ of $2 \mathrm{M} \mathrm{H}_{2} \mathrm{SO}_{4}$. The absorbance of wells were read at $480 \mathrm{~nm}$. The assays were performed twice and specific binding of serum antibody to anti-CAA I or anti-CAA II was determined by subtraction of the average absorbance of control wells (uncoated with CA) from that of antigen coated wells.

\section{Determination of malondialdehyde (MDA)} levels in erythrocytes: The MDA levels in erythrocytes were determined as described in a previous publication (Agilent 1100 series HPLC systems, Waldbronn, Germany), C18 column was used for measurements (ZORBAX Eclipse XDB-C18; $4.6 \times 150 \mathrm{~mm} ;$ Agilent Technologies, Santa Clara, CA, USA) [12]. Detection was performed fluorometrically (excitation $536 \mathrm{~nm}$ and emission $555 \mathrm{~nm}$ ). Tetraethoxypropane was utilized as MDA standard $(1.25-0.035 \mathrm{Mm})$ and results were represented as $\mathrm{mM} / \mathrm{g} \mathrm{Hb}$.

Determination of catalase (CAT) activity: The catalase activity in erythrocyes was determined by the method as described in relevant literature
[13]. This method relies on the fact that the absorbance at $240 \mathrm{~nm}$ is diminished due to the dismutation of $\mathrm{H}_{2} \mathrm{O}_{2}$ and results are demonstrated as $\mathrm{k} / \mathrm{g} \mathrm{Hb}$ ( $\mathrm{k}$, rate constant)

\section{Determination of superoxide dismutase (SOD)} activity: The SOD activity in erythrocytes was assessed by the method as described in a previous publication [14]. Formazan formation was evaluated spectrophotometrically at 560 $\mathrm{nm}$ and results were shown as $\mathrm{U} / \mathrm{g} \mathrm{Hb}$.

\section{Determination of glutathione peroxidase} (GPx) activity: The activity of GPx was measured with a spectrophotometric assay kit (Assay Desings, Ann Arbor, MI, USA). In this kit, the results were expressed as U/g Hb.

\section{Statistical analysis}

Data derived from the results of this study was analyzed using IBM SPSS Statistics 20 program. The normal distribution of data was assessed using Kolmogorov-Smirnov test. A comparative analysis of the erythrocyte superoxide dismutase, catalase, and glutathione peroxidase and malondialdehyde levels was carried out via the independent samples T-test. Mann-Whitney U was employed for the other non-parametric comparisons. Correlation between variables was tested using Pearson's correlation test. The level of significance was set at $\mathrm{p}$ value $<0.05$.

\section{Results}

As indicated in Figure 1 and Table 1, serum anti-CAA I titers did not differ between endometriosis and control groups $(0.39 \pm 0.12$ vs. $0.37 \pm 0.08$; respectively, $p=0.41$ ). On the other hand, anti-CAA II titers were remarkably increased in endometriosis patients than that in controls $(0.30 \pm 0.11$ vs. $0.241 \pm 0.080 ; p=0.027)$ (Table 1). The absorbance values $>0.401$, the 
mean absorbance \pm 2 SD of control subjects, were termed as positive. The positive results were noted in 7 of 33 endometriosis cases (Figure 2).

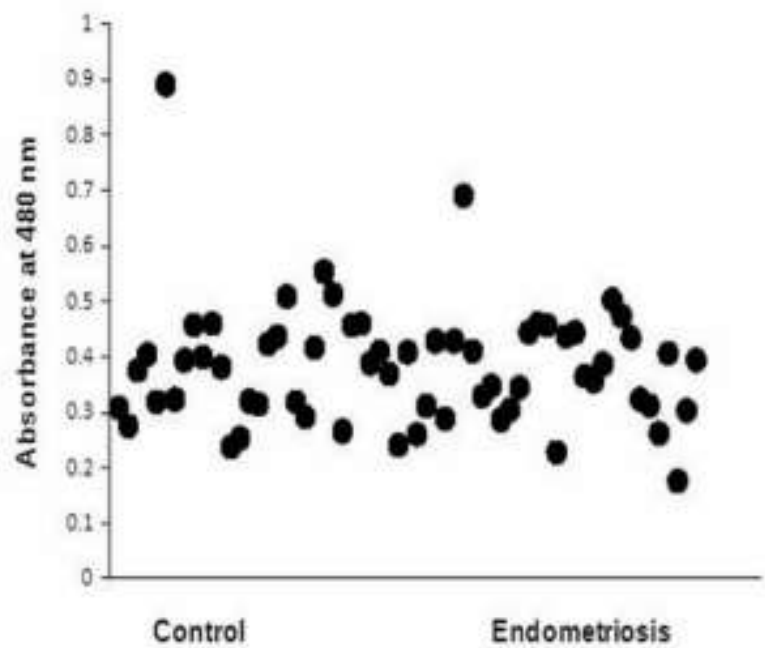

Figure 1. Anti CAA I in sera from endometriosis patients and control subjects.

Table 1. Serum levels of oxidative stress markers and anti-carbonic anhydrase antibodies in control and endometriosis groups.

\begin{tabular}{|l|l|l|l|}
\hline \multirow{2}{*}{ Variable } & \multicolumn{2}{|c|}{ Groups } & \multicolumn{1}{c|}{$\begin{array}{c}p \\
\text { Value }\end{array}$} \\
\cline { 2 - 4 } & Control & Endometriosis & \\
\hline $\begin{array}{l}\text { Anti-CAA I } \\
\text { (absorbance) }\end{array}$ & $0.392 \pm 0.12$ & $0.378 \pm 0.98$ & 0.41 \\
\hline $\begin{array}{l}\text { Anti-CAA II } \\
\text { (absorbance) }\end{array}$ & $0.241 \pm 0.08$ & $0.300 \pm 0.11$ & $0.027^{*}$ \\
\hline SOD (U/g Hb) & $50.99 \pm 18.99$ & $62.43 \pm 32.51$ & 0.29 \\
\hline CAT (k/g Hb) & $68.51 \pm 39.52$ & $80.85 \pm 39.31$ & 0.24 \\
\hline GPx (U/g Hb) & $1.50 \pm 0.38$ & $1.67 \pm 0.24$ & $0.038^{*}$ \\
\hline $\begin{array}{l}\text { MDA (mM/g } \\
\text { Hb) }\end{array}$ & $5.50 \pm 0.68$ & $6.03 \pm 1.14$ & $0.037^{*}$ \\
\hline
\end{tabular}

Values: Mean $\pm S D ; A n t i-C A A=$ Anti-carbonic anhydrase antibodies; $S O D=$ Superoxide dismutase; $C A T=$ Catalase; $G P x=$ Glutathione peroxidase; $M D A=$ Malondialdehyde; $*=$ statistically significant.

The antioxidant enzyme activities (SOD, CAT and GPx) and MDA levels in erythrocytes were increased in endometriosis group; but only GPx activity and MDA levels were significantly higher in endometriosis group $(p=0.038$, and $p=0.037$, respectively) (Table 1 ). As shown in Table 2, no correlation was detected between anti-CAA I and II titers and SOD, CAT and GPx activities and MDA levels.

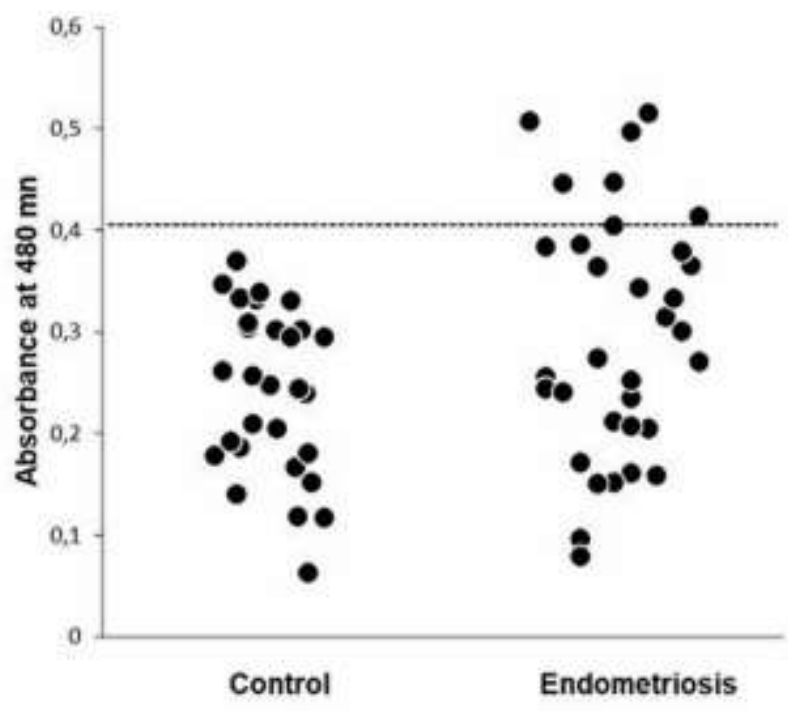

Figure 2. Anti-CAA II in sera from endometriosis patients and control subjects. The line indicates the mean value $\pm 2 \mathrm{SD}$ of control subjects $(\mathrm{A} 480=$ 0.401).

Table 2. Correlation analysis of anti-carbonic anhydrase antibodies to oxidative stress markers in the endometriosis patients.

\begin{tabular}{|l|l|l|l|}
\hline \multicolumn{2}{|l|}{ Variable } & $\begin{array}{l}\boldsymbol{r} \\
\text { Value }\end{array}$ & \multicolumn{1}{c|}{$\begin{array}{c}\boldsymbol{p} \\
\text { Value }\end{array}$} \\
\hline \multirow{4}{*}{ Anti-CAA I } & SOD & -0.205 & 0.148 \\
\cline { 2 - 4 } & CAT & -0.072 & 0.346 \\
\cline { 2 - 4 } & GPx & 0.030 & 0.435 \\
\cline { 2 - 4 } Anti-CAA II & MDA & -0.127 & 0.244 \\
& SOD & -0.006 & 0.488 \\
\cline { 2 - 4 } & CAT & 0.163 & 0.183 \\
\cline { 2 - 4 } & GPx & -0.209 & 0.122 \\
\cline { 2 - 4 } & MDA & 0.037 & 0.426 \\
\hline
\end{tabular}

Anti-CAA= Anti-carbonic anhydrase antibodies; $S O D=$ Superoxide dismutase; $C A T=$ Catalase; GPX= Glutathione peroxidase; MDA= Malondialdehyde; *= statistically significant. 


\section{Discussion}

The present study was performed to assess endometriosis patients in terms of serum levels of anti-CAA I, anti-CAA II and erythrocyte oxidative stress markers. Our results have shown that in subjects with endometriosis antiCAA II frequency [21\%] and erythrocytes oxidative stress markers were higher than control subjects (Figure 2 and Table 1). We concluded that there is an increased immune response to CA II in endometriosis patients. Increased erythrocyte oxidative stress observed in endometriosis may be effective in the mechanism of CA II autoantibody. These findings were similar to previous results reported in patients with polycystic ovary syndrome (26\%), systemic lupus erythematosus (31.2\%), Sjögren's Syndrome (17\%), and rheumatoid arthritis $(27.8 \%)[7,8,15,16]$.

Endometriosis was encountered in approximately $20-50 \%$ of infertile women that underwent laparoscopy during their infertility workup [2]. Since alteration of humoral and cellular immune systems is a feature of endometriosis, new diagnostic measures have focused on immunologic parameters related to endometriosis [17]. Elevated levels of ROS were previously reported to be associated with endometriosis [18,19]. Stimulation and improvement of mononuclear phagocytes lead to oxidative stress which causes a localized pelvic inflammatory process [20]. Szczepańska et al. reported a noteworthy reduction of SOD and GPx activities in peritoneal cavity with endometriosis [21]. In addition, it has been reported that oxidative stress observed in endometriosis also affects red blood cells [4].

Our results indicate that erythrocyte GPx activity and MDA levels were significantly higher in endometriosis patients compared to controls. It must be remembered that there is a critical interaction between oxidative stress and cytokines, chemokines and antioxidants. The levels of all of the antioxidants may not be necessarily altered significantly in order to eliminate the hazardous effects of oxidative stress. In addition, their levels may not always reflect the changes in the microenvironment influenced directly by the pathology. All in all, whether and to what extent oxidative stress is involved in endometriosis remain to be elucidated in further trials.

Carbonic anhydrases possess a serious function in gas transportation, acid-base balance and multiple secretion tasks in tissues [22]. The endometrium and placenta are well-known sources of CA-I and CA-II suggesting a possible role of these enzymes in fertilization and fetoplacental development [23]. Since CA I and CA II are the most frequent human isoenzymes, immunoglobulin $\mathrm{G}$ autoantibodies formed against CA I and CA II have been identified in various autoimmune diseases $[15,16,24,25]$. In our study, serum levels of only anti-CAA II were significantly increased in women with endometriosis.

The way of antibody formation against CA II has not yet been clearly elucidated, however, protein modifications and oxidative imbalance are important reasons for autoimmunity. Fuji et al. has reported that elevated oxidative stress in erythrocytes causes antibody production against CA II [11]. The final products of lipid peroxidation such as HNE (4-hydroxy-2nonenal) and MDA bind to proteins and change their antigenic features. One of the major targets of these end-products in red blood cells is CA II [26]. In case of erythrocytes in endometriosis are prone to oxidative stress, CA II may gain an antigenic character as a result of similar alterations. In an experimental study, anti-CAA II was found to inhibit the enzymatic activity of CA [27]. 
From our results, we understood that anti-CAA II, rather than anti-CAA I, seem to be involved in endometriosis. Identification and discrimination of CA I and CA II in terms of functional and physiological impacts can provide a better understanding. We found no correlation between oxidative stress markers and autoantibodies against carbonic anhydrase enzymes. The complex interaction between immune system and antioxidant mechanisms constitute a challenge for researchers to make more accurate and precise interpretations. Further attempts must be directed to explore the functions of subgroups of antibodies and antioxidant systems individually.

\section{Conclusion}

Erythrocyte oxidative stress and anti-CAA II may be associated with the pathophysiology of endometriosis. Taking the complexity of inflammatory reactions into account, the beneficial effects of oxidative stress balance and immunomodulation need to be investigated further using larger study groups.

Funding: There is no financial support and sponsorship

Conflict of Interest: The authors declare that they have no conflict of interest.

Ethical statement: The study was reviewed and approved by the local ethics committee (Date and Decision no: 17/04/2014, 2014/65).

ORCID iD of the author(s)

Ulku Mete Ural / 0000-0002-2176-7863

Ahmet Alver / 0000-0002-9617-6689

Ayse Senturk / 0000-0002-3542-2153

Imran Ince / 0000-0003-2232-3444

Beril Gurlek / 0000-0002-4050-3193

\section{References}

[1]Macer ML, Taylor HS. Endometriosis and infertility: a review of the pathogenesis and treatment of endometriosis-associated infertility. Obstet Gynecol Clin North Am. 2012;39(4):535-49.

[2]Gupta S, Agarwal A, Krajcir N, et al. Role of oxidative stress in endometriosis. Reprod Biomed Online. 2006;13(1):126-34.

[3]Yeaman GR, Collins JE, Lang GA. Autoantibody responses to carbohydrate epitopes in endometriosis. Ann N Y Acad Sci. 2002;955:174-82.

[4]Andrisani A, Donà $G$, Brunati AM, et al. Increased oxidation-related glutathionylation and carbonic anhydrase activity in endometriosis. Reprod Biomed Online. 2014;28(6):773-79.

[5]Lang GA, Yeaman GR. Autoantibodies in endometriosis sera recognize a ThomsenFriedenreich-like carbohydrate antigen. J Autoimmun. 2001;16(2):151-61.

[6]Supuran CT, Scozzafava A. Carbonic anhydrases as targets for medicinal chemistry. Bioorg Med Chem. 2007;15(13):4336-50.

[7]Pertovaara M, Parkkila S, Korpela M. Anticarbonic anhydrase autoantibodies and serum beta- 2 microglobulin correlate with the ClinESSDAI score in patients with Sjögren's syndrome. Clin Exp Rheumatol. 2017;35(2):351.

[8]Alver A, Senturk A, Cakirbay H, et al. Carbonic anhydrase II autoantibody and oxidative stress in rheumatoid arthritis. Clin Biochem. 2011;44(17-18):1385-89.

[9]Alver A, Mentese A, Karahan SC. Increased Serum Anti-Carbonic Anhydrase II Antibodies in patients with Graves ' disease. Exp Clin Endocrinol. 2007;115(5):287-91.

[10] Adamus G, Yang S, Weleber RG. Unique epitopes for carbonic anhydrase II 
autoantibodies related to autoimmune retinopathy and cancer-associated retinopathy. Exp Eye Res. 2016;147:161168.

[11]Iuchi Y, Okada F, Onuma K, et al. Elevated oxidative stress in erythrocytes due to SOD1 deficiency causes anemia and triggers autoantibody production. Biochem J. 2007;402(2):219-27.

[12] Alver A, İmamoğlu M, Menteşe A, et al. Malondialdehyde and CA II autoantibody levels are elevated in children with undescended testes. World J Urol. 2014;32(1):209-13.

[13]Carl H, Soumya R, Srinivas P, et al. Oxidative stress in erythrocytes of banked ABO blood. Hematology. 2016;21(10):63034.

[14] Ansari FA, Ali SN, Mahmood R. Sodium nitrite-induced oxidative stress causes membrane damage, protein oxidation, lipid peroxidation and alters major metabolic pathways in human erythrocytes. Toxicol In Vitro. 2015;29(7):1878-86.

[15]Caccavo D, Afeltra A, Rigon A, et al. Antibodies to carbonic anhydrase in patients with connective tissue diseases: relationship with lung involvement. Int J Immunopathol Pharmacol. 2008;21(3):659-67.

[16] Mentese A, Guven S, Sumer A, et al. Serum anti-carbonic anhydrase I and II antibodies and polycystic ovary syndrome. Turk J Biochem. 2013;38:43-48.

[17] Liu F, He L, Liu Y, et al. The expression and role of oxidative stress markers in the serum and follicular fluid of patients with endometriosis. Clin Exp Obstet Gynecol. 2013;40(3):372-76.

[18] Singh AK, Chattopadhyay R, Chakravarty $\mathrm{B}$, et al. Markers of oxidative stress in follicular fluid of women with endometriosis and tubal infertility undergoing IVF. Reprod Toxicol. 2013;42:116-24.

[19]Donnez J, Binda MM, Donnez O, et al. Oxidative stress in the pelvic cavity and its role in the pathogenesis of endometriosis. Fertil Steril. 2016;106(5):1011-17.

[20] Prieto L, Quesada JF, Cambero O, et al. Analysis of follicular fluid and serum markers of oxidative stress in women with infertility related to endometriosis. Fertil Steril. 2012;98(1):126-30.

[21] Szczepańska M, Koźlik J, Skrzypczak J, et al. Oxidative stress may be a piece in the endometriosis puzzle. Fertil Steril. 2003;79(6):1288-93.

[22] Supuran CT. Structure and function of carbonic anhydrases. Biochem J. 2016;473(14):2023-32.

[23]Reimand K, Talja I, Metsküla K, et al. Autoantibody studies of female patients with reproductive failure. J Reprod Immunol. 2001;51(2):167-76.

[24]Hosoda H, Okawa-Takatsuji M, Tanaka A, et al. Detection of autoantibody against carbonic anhydrase II in various liver diseases by enzyme-linked immunosorbent assay using appropriate conditions. Clin Chim Acta. 2004;342(1-2):71-81.

[25] Alver A, Menteşe A, Menteşe Ü, et al. Anticarbonic anhydrase II antibodies in endstage renal disease patients. Med Princ Pract. 2014;23(4):331-5.

[26] Toyoda K, Nagae R, Akagawa M. Proteinbound 4-hydroxy-2-nonenal: an endogenous triggering antigen of anti- DNA response. $\mathbf{J}$ Biol Chem. 2007;282(35):25769-78.

[27] Botrè F, Botrè C, Podestà E, et al. Effect of anti-carbonic anhydrase antibodies on carbonic anhydrases I and II. Clin Chem. 2003;49(7):1221-23. 